\title{
A quantum-enhanced precision medicine application to support data-driven clinical decisions for the personalized treatment of advanced knee osteoarthritis: development and preliminary validation of precisionKNEE_QNN
}

\author{
Nima Heidari,${ }^{1,2,5}$ Stefano Olgiati, ${ }^{*}, 2,3,4,5$ Davide Meloni, ${ }^{4}$ Federico Pirovano, ${ }^{4}$ \\ Ali Noorani, ${ }^{2,5}$ Mark Slevin ${ }^{1}$ and Leonard Azamfirei ${ }^{1}$

\footnotetext{
${ }^{1}$ George Emil Palade University of Medicine, Pharmacy, Science and Technology, Department of Anesthesiology and Intensive Care, Targu Mures 540139, Romania, ${ }^{2}$ nextAI, E10 5NP London, United Kingdom, ${ }^{3}$ University of Ferrara, Department of Medical Biotechnologies, 44121 Ferrara, Italy, ${ }^{4}$ QuantumSPEKTRAL, 20100 Milan, Italy and ${ }^{5}$ Orthopaedic Specialists Artificial Intelligence Unit (OS-AI Unit), W1G 8HU, London, United Kingdom

*Corresponding author. stefano.olgiati@unife.it
}

\section{Abstract}

Background

Quantum computing (QC) and quantum machine learning (QML) are promising experimental technologies which can improve precision medicine applications by reducing the computational complexity of algorithms driven by big, unstructured, real-world data. The clinical problem of knee osteoarthritis is that, although some novel therapies are safe and effective, the response is variable, and defining the characteristics of an individual who will respond remains a challenge. In this paper we tested a quantum neural network (QNN) application to support precision data-driven clinical decisions to select personalized treatments for advanced knee osteoarthritis.

Methods

Following patients' consent and Research Ethics Committee approval, we collected clinico-demographic data before and after the treatment from 170 patients eligible for knee arthroplasty (Kellgren-Lawrence grade $\geq 3$, OKS $\leq 27$, Age $\geq 64$ and idiopathic aetiology of arthritis) treated over a 2 year period with a single injection of microfragmented fat. Gender classes were balanced $(76 \mathrm{M}, 94 \mathrm{~F})$ to mitigate gender bias. A patient with an improvement $\geq 7$ OKS has been considered a Responder. We trained our QNN Classifier on a randomly selected training subset of 113 patients to classify responders from non-responders (73 R, $40 \mathrm{NR}$ ) in pain and function at 1 year. Outliers were hidden from the training dataset but not from the validation set.

Results

We tested our QNN Classifier on a randomly selected test subset of 57 patients (34 R, 23 NR) including outliers. The No Information Rate was equal to 0.59. Our application correctly classified 28 Responders out of 34 and 6 non-Responders out of 23 (Sensitivity $=0.82$, Specificity $=0.26$, F1 Statistic $=0.71)$. The Positive $(\mathrm{LR}+)$ and Negative (LR-) Likelihood Ratios were respectively 1.11 and 0.68. The Diagnostic Odds Ratio (DOR) was equal to 2.

Conclusions

Preliminary results on a small validation dataset show that quantum machine learning applied to data-driven clinical decisions for the personalized treatment of advanced knee osteoarthritis is a promising technology to reduce computational complexity and improve prognostic performance. Our results need further research validation with larger, real-world unstructured datasets, and clinical validation with an AI Clinical Trial to test model efficacy, safety, clinical significance and relevance at a public health level.

Key words: Quantum Machine Learning, Quantum Neural Network Classifiers, Digital Medicine, Data-driven Precision Medicine, Knee Osteoarthritis, Biologics

NOTE: This preprint reports new research that has not been certified by peer review and should not be used to guide clinical practice. 


\section{BACKGROUND}

Applied research has shown that quantum computing applied to machine learning is a novel experimental digital medicine technology [Cordier, Sawaya, Guerreschi, and McWeeney, 2021] which can reduce computational complexity [Sengupta and Srivastava, 2021, Singh and Aarthi, 2021] and improve data-driven clinical decisions [Solenov, Brieler, and Scherrer, 2018].

A number of biologic therapies, including the injection of Microfragmented Adipose Tissue, have been shown to be safe and effective [Heidari, Noorani, Slevin, Cullen, Stark, Olgiati, Zerbi, and Wilson, 2020, Borg, Heidari, Noorani, Slevin, Cullen, Olgiati, Zerbi, Danovi, and Wilson, 2021, Heidari, Borg, Olgiati, Slevin, Danovi, Fish, Wilson, and Noorani, 2021a]. The outcomes of these treatments can be variable [Sihvonen, Paavola, Malmivaara, and Järvinen, 2013] with some patients showing dramatic improvements in their symptoms whilst others fail to respond [Borg, Heidari, Noorani, Slevin, Cullen, Olgiati, Zerbi, Danovi, and Wilson, 2021].

Defining the characteristics of individual who will respond remains a challenge. Applications and tools to support clinical decisions with predictive, data-driven, precision medicine can identify patients who will respond with reduction in pain and improvement in function to novel as well as established treatments based on pre-treatment clinico-demographic data [Heidari, Parkin, Olgiati, Meloni, Fish, Noorani, Slevin, and Azamfirei, 2021b]. This approach is key in developing personalized, evidence-based clinical pathways.

\section{METHODS}

\section{Ethics Statement}

This study was carried out in compliance with the rules of the Helsinki Declaration and International Ethical Regulations [Association et al., 2009], including all subsequent amendments, under the approval of the Research Ethics Committee of the George Emil Palade University of Medicine, Pharmacy, Science and Technology of Targu Mures, Romania (research approval number 1464/2021).

\section{Dataset}

Following patients' consent and Research Ethics Committee approval, we collected clinico-demographic data before and after the treatment from 170 patients eligible for knee arthroplasty (Kellgren-Lawrence grade $\geq 3$, OKS $\leq 27$, Age $\geq 64$ and idiopathic aetiology of arthritis) treated over a 2 year period with a single injection of microfragmented fat. (Table 1)

Gender classes were balanced $(76 \mathrm{M}, 94 \mathrm{~F})$ to mitigate gender bias. A patient with an improvement $\geq 7$ OKS has been considered a Responder.

\section{Oxford Knee Score (OKS)}

OKS comprises of 12 questions that are scored 0-4 with 0 being severe compromise and 4 being no compromise, covering pain and function of the knee. The best outcome is a score of 48 and the worst score possible is 0 . This is a validated score or the measure of functional outcomes in patients undergoing knee arthroplasty. All 170 patients completed questionnaires before and at three months, six months and one-year following treatment [Dawson, Fitzpatrick, Murray, and Carr, 1998].

\section{Kellgren-Lawrence method of classifying the severity of osteoarthritis (OA)}

The Kellgren and Lawrence system is a common method of classifying the severity of osteoarthritis (OA) using five grades [Kohn, Sassoon, and Fernando, 2016].

- grade 0 (none): definite absence of $x$-ray changes of osteoarthritis

- grade 1 (doubtful): doubtful joint space narrowing and possible osteophytic lipping

- grade 2 (minimal): definite osteophytes and possible joint space narrowing

- grade 3 (moderate): moderate multiple osteophytes, definite narrowing of joint space and some sclerosis and possible deformity of bone ends

- grade 4 (severe): large osteophytes, marked narrowing of joint space, severe sclerosis and definite deformity of bone ends

Osteoarthritis is deemed present at grade 2 although of minimal severity 1.

\begin{tabular}{rrrr}
\hline & $\begin{array}{r}\text { Age on } \\
\text { Procedure }\end{array}$ & $\begin{array}{r}\text { Pre-operative } \\
\text { OKS }\end{array}$ & KL Grade \\
\hline mean & 74 & 21 & 4 \\
std dev & 7 & 6 & 0.4 \\
min & 65 & 0 & 3 \\
$\mathbf{2 5 \%}$ & 69 & 18 & 4 \\
$\mathbf{5 0 \%}$ & 72 & 21 & 4 \\
$\mathbf{7 5 \%}$ & 77 & 25 & 4 \\
$\mathbf{m a x}$ & 92 & 27 & 4 \\
\hline
\end{tabular}

Table 1. Baseline distribution of Age on Procedure, Preoperative Oxford Knee Score, and Kellgren-Lawrence grade of knee osteoarthritis. Source Dataset 1464/2021

\section{Model and Circuit Description}

We applied the approach to quantum problem solving developed by Zickert [2021]. We utilized a Parameterized Quantum Circuit (PQC) based on Quantum Machine Learning Neural Networks Classifier (QNN) technology developed by IBM and Qiskit Machine Learning. More specifically, we utilized OpflowQNN which is a neural network based application for the evaluation of quantum mechanical observables. OpflowQNN circuit is described in Fig. 1. where:

- $\mathrm{q}_{-} 0$ = qubit initialization

- $\mathrm{Z}, \mathrm{X}=$ Pauli $\mathrm{Z}$ and $\mathrm{X}$ gates

- $\mathrm{H}=$ Hadamard gate

- $\mathrm{Ry}=$ single-qubit rotation gate through angle $\theta$ (radians) around the y-axis.

- $\mathrm{Rx}=$ single-qubit rotation gate through angle $\theta$ (radians) around the $\mathrm{x}$-axis

\section{Copyright Notice}

Our code is an alteration of Qiskit Machine Learning which is a Copyright of IBM 2017, 2021. Qiskit is licensed under the Apache License, Version 2.0. All modifications of the code and derivative works are the responsibility of the authors.

\section{Model training}

We trained our QNN Classifier on a randomly selected training subset of 113 patients treated over a 2 year period with a 
medRxiv preprint doi: https://doi.org/10.1101/2021.12.13.21267704; this version posted December 14,2021 . The copyright holder for this

preprint (which was not certified by peer review) is the author/funder, who has granted medRxiv a license to display the preprint in perpetuity.

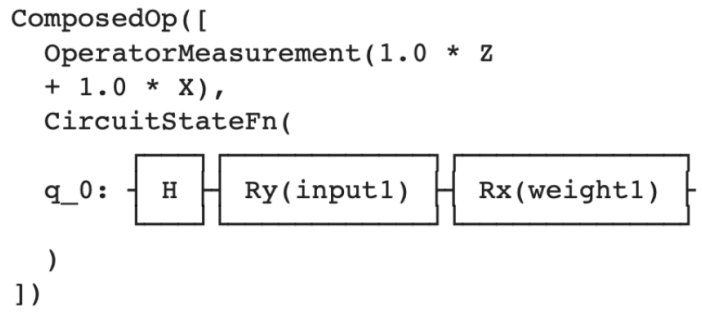

Fig. 1. Circuit Description. Source: IBM, Qiskit and OpflowQNN.

single injection of microfragmented fat classified as responders (R; OKS improvement $\geq 7$ ) and non-responders (R; OKS improvement $<7)$ in pain and function at 1 year $(73 \mathrm{R}, 40$ NR). Outliers were hidden from the training dataset but not from the validation set. (Fig. 2)

We initialized a 2-qubit Aer Simulator Backend with 1024 shots. We utilized a "Constrained Optimization BY Linear Approximation" (COBYLA) optimizer to minimize the Objective Function Value. (Fig. 3)

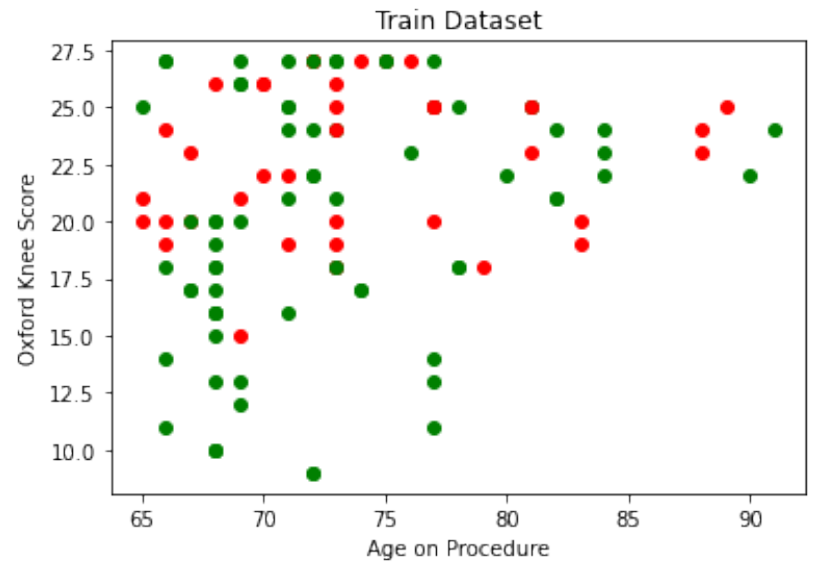

Fig. 2. $\mathrm{X}$-axis $=$ Age on Procedure $\mathrm{Y}$-Axis $=$ Pre-operative Oxford Knee Score; Green dots $=$ Responders with an improvement of $\geq 7$ points in the Oxford Knee Score at Year 1; Red dots = Non-Responders with an improvement of $<7$ points in the Oxford Knee Score at Year 1. Source Dataset $1464 / 2021$.

\section{Model validation}

The application has been tested on a balanced raw dataset including outliers of 57 patients ( $34 \mathrm{R}, 23 \mathrm{NR}$ ) with KellgrenLawrence grade $=3$ and 4 , OKS $\leq 27$, Age $\geq 64$ and idiopathic aetiology of arthritis. (Fig. 4, Table 3)

\section{RESULTS}

\section{Model Results}

Our application correctly classified 28 Responders out of 34 and 6 non-Responders out of 23 (Sensitivity $=0.82$, Specificity $=$ 0.26 , F1 Statistic $=0.71)$. The Positive $(\mathrm{LR}+)$ and Negative

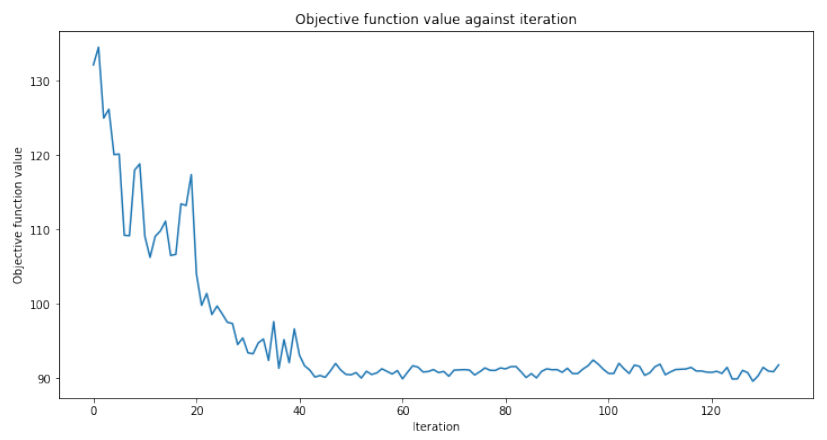

Fig. 3. Constrained Optimization BY Linear Approximation11 (COBYLA) optimizer to minimize the Objective Function Value. Source: IBM, Qiskit and OpflowQNN.

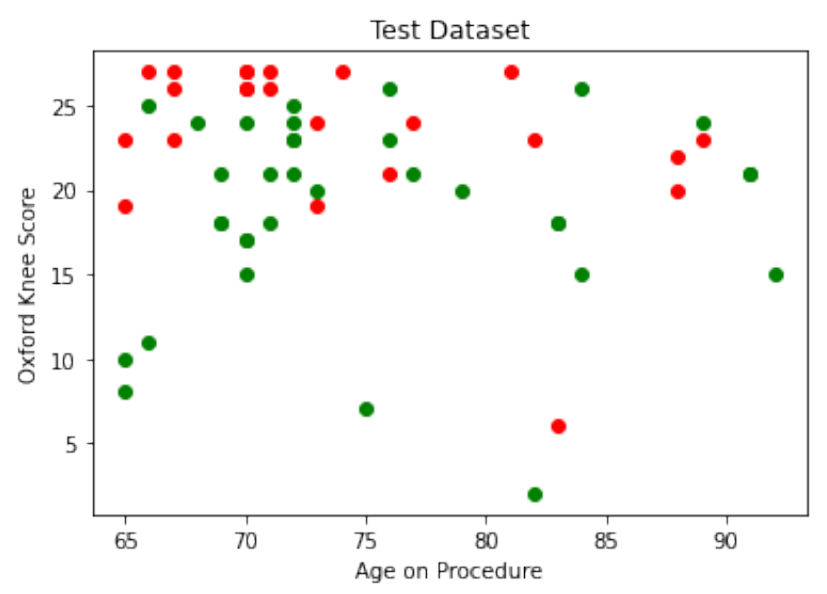

Fig. 4. $\mathrm{X}$-axis $=$ Age on Procedure $\mathrm{Y}$-Axis $=$ Pre-operative Oxford Knee Score; Green dots $=$ Responders with an improvement of $\geq 7$ points in the Oxford Knee Score at Year 1; Red dots = Non-Responders with an improvement of $<7$ points in the Oxford Knee Score at Year 1. Source Dataset 1464/2021.

(LR-) Likelihood Ratios were respectively 1.11 and 0.68 . The Diagnostic Odds Ratio was equal to 2. (Table 2 and Fig. 5)

\begin{tabular}{|c|c|c|c|c|}
\cline { 3 - 5 } \multicolumn{2}{c|}{} & \multicolumn{3}{|c|}{ Pred } \\
\cline { 3 - 5 } \multicolumn{2}{c|}{} & R & NR & Obs \\
\hline \multirow{3}{*}{ Obs } & R & 28 & 6 & 34 \\
\cline { 2 - 5 } & NR & 17 & 6 & 23 \\
\cline { 2 - 5 } & Pred & 45 & 12 & 57 \\
\hline
\end{tabular}

Table 2. Confusion Matrix. Source precisionKNEE_QNN applied to Test Set from Dataset 1464/2021

\section{Model Interpretation}

The use of the precisionKNEE_QNN clinical decision-making tool requires external validation. This is a new technology best suited to large unstructured datasets. Some of traditional methods of validation may not be appropriate for Quantum Machine Learning tools such as ours [Hidary, 2019].

The SPIRIT-AI [Rivera, Liu, Chan, Denniston, and Calvert, 2020]) and CONSORT- AI [Liu, Rivera, Moher, Calvert, and Denniston, 2020] initiatives provide guidance to improve the transparency and completeness of reporting of clinical trials 


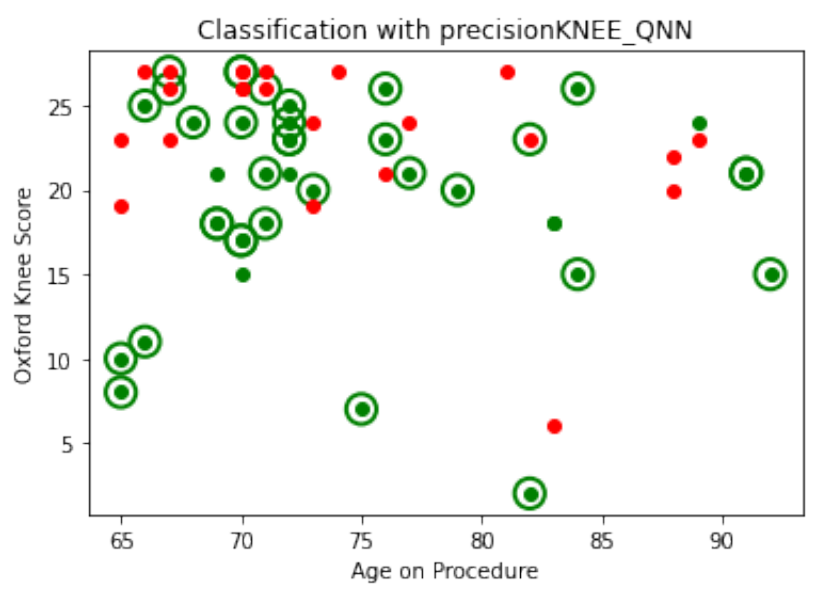

Fig. 5. $\mathrm{X}$-axis $=$ Age on Procedure $\mathrm{Y}$-Axis $=$ Pre-operative Oxford Knee Score; Green dots $=$ Responders with an improvement of $\geq 7$ points in the Oxford Knee Score at Year 1; Red dots = Non-Responders with an improvement of $<7$ points in the Oxford Knee Score at Year 1; Green Circles = Correct classification of either Responders or NonResponders; No Circles = Incorrect classification of either Responders or Non-Responders. Source Dataset 1464/2021.

\begin{tabular}{ll}
\hline Metric & Value \\
\hline Sensitivity & 0.8235 \\
\hline Specificity & 0.2609 \\
\hline LR+ & 1.1142 \\
\hline LR- & 0.6765 \\
\hline DOR & 2.0000 \\
\hline F1 & 0.7088 \\
\hline
\end{tabular}

Table 3. Test Performance Metrics. Source precisionKNEE_QNN applied to Test Set from Dataset 1464/2021

evaluating interventions involving artificial intelligence. The utility of these initiatives is not clear for use in Quantum Machine Learning.

\section{Limitations}

Despite being one of the largest datasets of its kind, upon stratification we observe groups of patients with minimal observations and thus we cannot conclude external validity in the findings pertaining to these groups. Kellgren-Lawrence classification of arthritis was found to be an important feature during modelling. This measure is crude and correlates poorly with patient symptoms.

MFAT is the only treatment used in our model. With inclusion of other treatment modalities, a true clinical decisionmaking support tool can be developed. Patients find these very useful and by providing a personalised prediction of the possible outcome, they will be able to play a more meaningful role in the decision-making process.

\section{CONCLUSIONS}

Preliminary results on a small validation dataset show that quantum machine learning applied to data-driven clinical decisions for the personalized treatment of advanced knee osteoarthritis is a promising technology to reduce computational complexity and improve prognostic performance.
Our results need further research validation with larger, real-world unstructured datasets, and clinical validation with an AI Clinical Trial to test model efficacy, safety, clinical significance and relevance at a public health level.

\section{AUTHOR CONTRIBUTIONS STATEMENT}

S.O. and N.H. were responsible for conceptualization; S.O. and N.H. were responsible for methodology; S.O. and D.M. were responsible for the software; S.O., F.P., and N.H. were responsible for the formal analysis; N.H. and A.N. were responsible for the investigation; N.H. was responsible for the resources; N.H. was responsible for data curation; N.H. and S.O. were responsible for writing?original draft preparation; N.H., S.O., D.M. and F.P. were responsible for writing?review and editing; N.H. and S.O. were responsible for visualization. All authors have read and agreed to the published version of the manuscript. S.O., and N.H. are responsible for the algorithms and processes on bias mitigation.

\section{COMPETING INTERESTS}

The authors declare that they have received support for the present manuscript in terms of provision of data and study materials. In the past 36 months, the authors have received grants from public research institutions and contracts from biomedical devices companies pertaining the field of digital health, digital medicine and osteoarthritis. The authors have received and are likely to receive in the future royalties, licenses, consulting fees, payment or honoraria for lectures, presentations, speakers' bureaus, manuscript writing or educational events. Some parts of the Quantum Circuits and Artificial Intelligence algorithms are copyrighted and the authors are likely to patent some of the contents. The authors hold stock and stock options directly and indirectly in publicly traded and private biotech, medtech, digital health and healthcare companies. The authors declare no other competing interests.

\section{ETHICS STATEMENT}

This study was carried out in compliance with the rules of the Helsinki Declaration and International Ethical Regulations, including all subsequent amendments, under the approval of the Research Ethics Committee of the "George Emil Palade" University of Medicine, Pharmacy, Science and Technology of Targu Mures, Romania (research approval number 1464/2021).

\section{ACKNOWLEDGMENTS}

The authors acknowledge Angela Cullen as the Data Manager and Analyst.

\section{ORCID}

- Nima Heidari (https://orcid.org/0000-0002-3532-1096)

- Stefano Olgiati (https://orcid.org/0000-0001-5181-8433)

- Mark Slevin (https://orcid.org/0000-0003-3767-4861)

- Leonard Azamfirei (https://orcid.org/0000-0003-3220-2267) 


\section{References}

Benjamin A Cordier, Nicolas PD Sawaya, Gian G Guerreschi, and Shannon K McWeeney. Biology and medicine in the landscape of quantum advantages. arXiv preprint arXiv:2112.00760, 2021.

Kinshuk Sengupta and Praveen Ranjan Srivastava. Quantum algorithm for quicker clinical prognostic analysis: an application and experimental study using ct scan images of covid-19 patients. BMC Medical Informatics and Decision Making, 21(1):1-14, 2021.

Paras Nath Singh and S Aarthi. Quantum circuits-an application in qiskit-python. In 2021 Third International Conference on Intelligent Communication Technologies and Virtual Mobile Networks (ICICV), pages 661-667. IEEE, 2021.

Dmitry Solenov, Jay Brieler, and Jeffrey F Scherrer. The potential of quantum computing and machine learning to advance clinical research and change the practice of medicine. Missouri medicine, 115(5):463, 2018.

Nima Heidari, Ali Noorani, Mark Slevin, Angela Cullen, Laura Stark, Stefano Olgiati, Alberto Zerbi, and Adrian Wilson. Patient-centered outcomes of microfragmented adipose tissue treatments of knee osteoarthritis: an observational, intentionto-treat study at twelve months. Stem Cells International, 2020, 2020.

Tiffanie-Marie Borg, Nima Heidari, Ali Noorani, Mark Slevin, Angela Cullen, Stefano Olgiati, Alberto Zerbi, Alessandro Danovi, and Adrian Wilson. Gender-specific response in pain and function to biologic treatment of knee osteoarthritis: a gender-bias-mitigated, observational, intention-to-treat study at two years. Stem Cells International, 2021, 2021.

Nima Heidari, Tiffanie-Marie Borg, Stefano Olgiati, Mark Slevin, Alessandro Danovi, Brady Fish, Adrian Wilson, and Ali Noorani. Microfragmented adipose tissue injection (mfat) may be a solution to the rationing of total knee replacement: A prospective, gender-bias mitigated, reproducible analysis at two years. Stem Cells International, 2021, 2021a.
Raine Sihvonen, Mika Paavola, Antti Malmivaara, and Teppo LN Järvinen. Finnish degenerative meniscal lesion study (fidelity): a protocol for a randomised, placebo surgery controlled trial on the efficacy of arthroscopic partial meniscectomy for patients with degenerative meniscus injury with a novel rct within-a-cohort study design. BMJ open, 3 (3):e002510, 2013.

Nima Heidari, James Michael Parkin, Stefano Olgiati, Davide Meloni, Brady Fish, Ali Noorani, Mark Slevin, and Leonard Azamfirei. A gender-bias-mitigated, data-driven precision medicine system to assist in the selection of biological treatments of grade 3 and 4 knee osteoarthritis: development and preliminary validation of precisionknee. medRxiv, 2021b.

World Medical Association et al. Declaration of helsinki. ethical principles for medical research involving human subjects. Jahrbuch Für Wissenschaft Und Ethik, 14(1):233-238, 2009.

Jill Dawson, Ray Fitzpatrick, David Murray, and Andrew Carr. Questionnaire on the perceptions of patients about total knee replacement. The Journal of bone and joint surgery. British volume, 80(1):63-69, 1998.

Mark D Kohn, Adam A Sassoon, and Navin D Fernando. Classifications in brief: Kellgren-lawrence classification of osteoarthritis. Clinical Orthopaedics and Related Researchß, 474(8):1886-1893, 2016.

Frank Zickert. Hands-on Quantum Machine Learning with Python. PyQML, 2021.

Jack D Hidary. Quantum Computing: An Applied Approach. Springer, 2019.

Samantha Cruz Rivera, Xiaoxuan Liu, An-Wen Chan, Alastair K Denniston, and Melanie J Calvert. Guidelines for clinical trial protocols for interventions involving artificial intelligence: the spirit-ai extension. bmj, 370, 2020.

Xiaoxuan Liu, Samantha Cruz Rivera, David Moher, Melanie J Calvert, and Alastair K Denniston. Reporting guidelines for clinical trial reports for interventions involving artificial intelligence: the consort-ai extension. bmj, 370, 2020. 